\title{
Validation of a new mobile phone dietary assessment tool DietPhone against diet diaries and urinary nitrogen
}

\author{
L. Monaghan, A. Paget, D. Young, K. Aitchison, H. I. M. Davidson and M. E. Clapham \\ Health Sciences, Queen Margaret University, Edinburgh EH21 6UU, UK
}

Dietary assessments methods are vital in assessing nutrient intakes in individuals and groups. New dietary assessment technologies are now becoming available, which may potentially improve the accuracy and efficiency of dietary assessment. However, limited amount of research has been performed to determine if new technologies are valid methods of dietary assessment.

DietPhone is an application downloadable onto mobile phone handsets. It has been developed and designed by staff at Queen Margaret University to record dietary intake. It contains the complete food database published by the FSA ${ }^{(1)}$.

To determine the relative validity of a new dietary assessment technology, the DietPhone, was compared to Diet Diary and using an independent marker of urinary nitrogen as a biomarker of validity.

Thirty adult subjects recorded their dietary intake for five consecutive week days using two forms of dietary assessment methods, the Dietphone and Diet Diary, and ten of these adults also completed a 24-h urine collection. The Bland-Altman ${ }^{(2)}$ methodology was used to compare the difference between the two methods, for several nutrients, to detect bias between methods. The Pearson correlation coefficient was also performed to determine the strength relationship between 24-h urinary nitrogen and protein intake.

Table. Comparing mean nutrient intake determined by Diet Diary and DietPhone

\begin{tabular}{lcccc}
\hline & Diet Diary $(\mathrm{DD})$ & DietPhone $(\mathrm{DP})$ & Mean difference $(\mathrm{DD}-\mathrm{DP})$ & 95\% CI \\
\hline Mean energy $(\mathrm{kJ})$ & 7077 & 6858 & 219 & -1191 to 1627 \\
Mean carbohydrate $(\mathrm{g})$ & 214 & 206 & 8 & -67 to 83 \\
Mean protein $(\mathrm{g})$ & 64.8 & 65.4 & 0.6 & -14.3 to 15 \\
Mean Fe (mg) & 9.7 & 9.6 & 0.2 & -2.1 to 2.7 \\
\hline
\end{tabular}

Both methods agreed at a group level for energy, carbohydrate, protein and Fe intake. However, a lack of agreement was found for these nutrients at an individual level.

A strong correlation was established for the two methods against 24-h urine protein, Diet Diary $r=0.70(P=0.024)$ and DietPhone $r=0.74(P=0.014)$.

This study has found that DietPhone strongly agrees with the Diet Diary at a group level. Both DietPhone and Diet Diary appear to determine protein intake to the same degree of accuracy when compared to an independent biomarker. Further development and research will be done to increase the validity of the Dietphone.

1. Food Standards Agency (2002) McCance and Widdowson's The Composition of Foods, 6th summary edition. Cambridge: Royal Society of Chemistry. 2. Bland MJ \& Altman DG (1999) Measuring agreement in method comparison studies. Statist Methods Med Res 8, 135-160. 